Pacific

Journal of

Mathematics

\title{
CLASSIFICATION OF SINGULARITIES \\ FOR A SUBCRITICAL FULLY NONLINEAR PROBLEM
}

MARÍA DEL MAR GONZÁLEZ 


\title{
CLASSIFICATION OF SINGULARITIES FOR A SUBCRITICAL FULLY NONLINEAR PROBLEM
}

\author{
MARÍA DEL MAR GONZÁLEZ
}

\begin{abstract}
We study isolated singularities for a fully nonlinear elliptic PDE of subcritical type. This equation appears in conformal geometry when dealing with the $k$-curvature of a locally conformally-flat manifold. (The $k$-curvature generalizes scalar curvature.) We give a classification result: either the function is bounded near the singularity, or it has a specific asymptotic behavior.
\end{abstract}

\section{Introduction}

The study of singularities for the subcritical problem

$$
-\Delta u=u^{\beta} \quad \text { in } B \backslash\{0\}, \quad \beta \in\left(\frac{n}{n-2}, \frac{n+2}{n-2}\right),
$$

has received a lot of attention. In particular, Gidas and Spruck [1981] gave a classification result: a positive solution of (1-1) with a nonremovable singularity at zero must behave like

$$
u(x)=(1+o(1)) \frac{c_{0}}{|x|^{2 /(\beta-1)}} \quad \text { near } x=0,
$$

for some $c_{0}=c_{0}(\beta, n)$. In this paper, we deal with a more general subcritical equation, of the form

$$
\sigma_{k}\left(A^{g_{v}}\right)=v^{\alpha} \quad \text { in } B \backslash\{0\}, \quad \alpha>0,
$$

where $g_{v}=v^{-2}|d x|^{2}$ for $v>0$ is a locally conformally-flat metric on the unit ball $B \subset \mathbb{R}^{n}$, with an isolated singularity at the origin. For a general metric $g$, the matrix $A^{g}$ is given by $A^{g}=g^{-1} \tilde{A}^{g}$, where $\tilde{A}^{g}$ is the Schouten tensor

$$
\tilde{A}_{i j}^{g}=\frac{1}{n-2}\left(R i c_{i j}-\frac{1}{2(n-1)} R g_{i j}\right)
$$

Keywords: subcritical problem, curvature equation, classification of singularities. 
while Ric and $R$ denote the Ricci tensor and the scalar curvature of $g$. In the metric $g_{v}$, the Schouten tensor becomes

$$
A^{g_{v}}=v\left(D^{2} v\right)-\frac{1}{2}|\nabla v|^{2} I .
$$

The curvatures $\sigma_{k}$ are defined as symmetric functions of the eigenvalues $\lambda_{1}, \ldots, \lambda_{n}$ of the (1,1)-tensor $A^{g}$,

$$
\sigma_{k}:=\sigma_{k}\left(A^{g}\right)=\sum_{i_{1}<\cdots<i_{k}} \lambda_{i_{1}} \cdots \lambda_{i_{k}} .
$$

The scalar curvature is simply

$$
\sigma_{1}=\lambda_{1}+\cdots+\lambda_{n}=\frac{1}{2(n-1)} R .
$$

Problem (1-2) for $k=1$ becomes the well known (1-1): if we write $u^{4 /(n-2)}=v^{-2}$ and $1+(n / 2)-\beta(n-2) / 2=\alpha$, the two problems are equivalent. Note that the critical exponent is $\beta=(n+2) /(n-2)$, or $\alpha=0$.

For a general $k$, we are dealing with a fully nonlinear equation of second order. The problem is elliptic in the positive cone

$$
\Gamma_{k}^{+}=\left\{v \mid \sigma_{1}\left(A^{g_{v}}\right), \ldots, \sigma_{k}\left(A^{g_{v}}\right)>0\right\},
$$

but, in general, not uniformly elliptic. However, it still carries an "almost" divergence structure

$$
m \sigma_{m}=v \partial_{j}\left(v_{i} T_{i j}^{m-1}\right)-n T_{i j}^{m-1} v_{i} v_{j}+\frac{n-m+1}{2} \sigma_{m-1}|\nabla v|^{2},
$$

where $T_{i j}^{m}$ denotes the Newton tensor (2-1). This was explored in [González 2005b].

Our main result is a classification of the isolated singularities of (1-2):

Theorem 1.1. Let $\alpha \in(0, k)$ and $n>2(k+1)$. If $v$ is a solution of

$$
\sigma_{k}(v)=v^{\alpha} \quad \text { in } B \backslash\{0\},
$$

with $v>0, v \in \Gamma_{k}^{+}$, and $v^{-1} \in \mathscr{C}^{3}(B \backslash\{0\})$, then

$$
v^{-1}(x) \leq \frac{C}{|x|^{2 k /(2 k-\alpha)}} \quad \text { near } x=0 .
$$

Theorem 1.2. Let $v$ be a solution of (1-3) for $\alpha \in(0,2 k /(k+1))$ and $n>2(k+1)$, with $v^{-1} \in \mathscr{C}^{3}(B \backslash\{0\})$. If the function $v^{-1}$ is not bounded near the origin, then there exist $c_{1}, c_{2}>0$ such that

$$
\frac{c_{1}}{|x|^{2 k /(2 k-\alpha)}} \leq v^{-1}(x) \leq \frac{c_{2}}{|x|^{2 k /(2 k-\alpha)}} \quad \text { near } x=0 .
$$


The local behavior of singularities for the critical problem $\sigma_{k}(v)=1$ has been addressed in [González 2005a]. There, we gave a sufficient condition for the function to be bounded near the singularity: the finiteness of the volume of the metric $g_{v}$ (when $n>2 k$ ). The same result was obtained by Han [2004] for $n=2 k$. For the Laplacian problem $(k=1)$, a complete classification of solutions was obtained by Caffarelli, Gidas, and Spruck [1989].

At the time this paper was submitted, it was conjectured that a similar classification result was true also for $\sigma_{k}$, where $n>2 k$. This has now been proved [Li 2006]. In the case $n<2 k$, all the singularities are removable [Gursky and Viaclovsky 2005].

One of the motivations for the study of (1-1) is that it appears in the resolution of the Yamabe problem (for a very good survey, see [Lee and Parker 1987]). We can establish an analogous $k$-Yamabe problem: find the infimum over all the metrics $g_{v}=v^{-2} g_{0}$ with $v>0$ of the functional

$$
\mathscr{F}_{k}(g)=\frac{1}{\operatorname{vol}(g)^{(n-2 k) / n}} \int_{M} \sigma_{k}\left(A^{g}\right) d \operatorname{vol}_{g} .
$$

This functional was first introduced by Viaclovsky [2000], and it generalizes the Yamabe functional. Its Euler equation is precisely $\sigma_{k}(v)=1$.

The global subcritical problem has been understood by Li and Li [2003]. Indeed, if $v$ is a positive solution of

$$
\sigma_{k}(v)=v^{\alpha} \quad \text { in } \mathbb{R}^{n}, \quad \alpha \geq 0,
$$

with $v^{-1} \in \mathscr{C}^{2}\left(\mathbb{R}^{n}\right)$, then either $v$ is constant, or $\alpha=0$ and

$$
v^{-1}(x)=\frac{a}{1+b^{2}|x-\bar{x}|^{2}}
$$

for some $\bar{x} \in \mathbb{R}^{n}$ and some positive constants $a$ and $b$.

The methods of Gidas and Spruck [1981] for the problem with $k=1$ can be generalized to our case. The key ingredient in the present paper is to understand the structure of $\sigma_{k}$ and, in particular, to replace the traceless Ricci tensor by the traceless $k$-Newton tensor (2-2).

The paper is structured as follows: in Section 2 we give some properties of $\sigma_{k}$ that will be crucial in the proofs. We use the divergence structure of $\sigma_{k}(2-5)$, an inductive process (2-7), and the properties of the traceless Newton tensor (2-2).

In Section 3 we establish the expression that will allow us to obtain the necessary $L^{p}$ estimates, through a generalization of an argument due to Obata and very successfully used by Chang, Gursky, and Yang [2002] and by Li and Li [2002]. In particular, we give a more refined formula (3-1) that is precisely the missing 
ingredient for the critical problem. The $L^{p}$ estimates are in Section 4, while in the last two sections we prove the theorems.

Remark 1.3. We believe that the theorems are also true for $n=2 k+1$, but, as in the case of [Gidas and Spruck 1981], one needs different estimates in (4-12).

Remark 1.4. We make the regularity assumption $v^{-1} \in \mathscr{C}^{3}(B \backslash\{0\})$. However, many of the arguments use integral estimates and only require that $v^{-1}$ is in some suitable Sobolev space; for instance, the whole of Section 4.

\section{Algebraic properties of $\sigma_{k}$}

For a general $n \times n$ matrix $A$, take its eigenvalues $\lambda_{1}, \ldots, \lambda_{n}$ and construct the symmetric functions $\sigma_{k}$, as well as the $k$-th Newton tensor

$$
T^{k}:=\sigma_{k}-\sigma_{k-1} A+\cdots+(-1)^{k} A^{k}=\sigma_{k} I-T^{k-1} A
$$

and the traceless Newton tensor

$$
L^{k}:=\frac{n-k}{n} \sigma_{k} I-T^{k} .
$$

Remark 2.1. Take $\sigma_{0}:=1$ and $T_{i j}^{0}:=\delta_{i j}$. Although the standard notation for a $(1,1)$-tensor is $A_{i}^{j}$, we write both indices as subindices without risking confusion.

Lemma 2.2 [Gårding 1959; Reilly 1973].

(1) $(n-k) \sigma_{k}=\operatorname{trace} T^{k}$;

(2) $(k+1) \sigma_{k+1}=\operatorname{trace}\left(A T^{k}\right)$;

(3) $\operatorname{trace} L^{k}=0$;

(4) if $\sigma_{1}, \ldots, \sigma_{k}>0$, then $T^{m}$ is positive definite for $m=1, \ldots k-1$;

(5) if $\sigma_{1}, \ldots, \sigma_{k}>0$, then $\sigma_{k} \leq C_{n, k}\left(\sigma_{1}\right)^{k}$.

In particular, if $A=A^{g_{v}}$ for $g_{v}=v^{-2}|d x|^{2}$, then the Schouten tensor becomes

$$
A_{i j}=v_{i j} v-\frac{1}{2}|\nabla v|^{2} \delta_{i j},
$$

while the traceless Ricci tensor (strictly speaking, a constant multiple of the actual traceless Ricci tensor) is now

$$
E_{i j}:=L_{i j}^{1}=v v_{i j}-\frac{1}{n} v \Delta v \delta_{i j} .
$$

Lemma 2.3 [Viaclovsky 2000]. Let $g_{v}=v^{-2}|d x|^{2}$. The Newton tensor $T^{m}$ for $m \leq n-1$ is divergence-free with respect to this metric; that is,

$$
\sum_{j} \tilde{\partial}_{j} T_{i j}^{m}=0, \quad \text { for all } i .
$$


As a consequence,

$$
\sum_{j} \tilde{\partial}_{j} L_{i j}^{m}=\frac{n-m}{n} \partial_{i} \sigma_{m}\left(A^{g_{v}}\right),
$$

where $\tilde{\partial}_{j}$ is the $j$-th covariant derivative with respect to the metric $g_{v}$, while $\partial_{j}$ denotes the usual Euclidean derivative.

The following two lemmas were proved in [González 2005b]. Expression (2-6) shows the 'almost' divergence structure of $\sigma_{m}$, while (2-7) is an inductive formula allowing us to handle the nondivergence terms (of order $m-1$ ) that appear in (2-6).

Lemma 2.4. In this setting,

$$
\begin{aligned}
& \sum_{j} \partial_{j} T_{i j}^{m}=-(n-m) \sigma_{m} v_{i} v^{-1}+n \sum_{i} T_{i j}^{m} v_{i} v^{-1} \quad \text { for each } i \\
& m \sigma_{m}\left(A^{g_{v}}\right)=v \sum_{i, j} \partial_{j}\left(v_{i} T_{i j}^{m-1}\right)-n \sum_{i, j} T_{i j}^{m-1} v_{i} v_{j}+\frac{n-m+1}{2} \sigma_{m-1}|\nabla v|^{2} .
\end{aligned}
$$

Lemma 2.5. Let $U$ be a domain in $\mathbb{R}^{n}, v^{-1} \in \mathscr{C}^{\infty}(U)$, and $\varphi \in \mathscr{C}_{0}^{\infty}(U)$ a smooth cutoff function. For any integers $1 \leq s \leq k \leq n$ and real number $\gamma$,

$$
\begin{aligned}
& \int_{U} \sum_{i, j} T_{i j}^{k-s} v_{i} v_{j}|\nabla v|^{2(s-1)} \varphi^{2 k} v^{-\gamma} d x \\
&=\left(1+\frac{k-s}{2 s}\right) \int_{U} \sigma_{k-s}|\nabla v|^{2 s} \varphi^{2 k} v^{-\gamma} d x \\
&+\frac{s+n+1-\gamma}{2 s} \int_{U} \sum_{i, j} T_{i j}^{k-s-1} v_{i} v_{j}|\nabla v|^{2 s} \varphi^{2 k} v^{-\gamma} d x \\
&-\frac{n-k+s+1}{4 s} \int_{U} \sigma_{k-s-1}|\nabla v|^{2(s+1)} \varphi^{2 k} v^{-\gamma} d x \\
&+\frac{k}{s} \int_{U} \sum_{i, j} T_{i j}^{k-s} v_{j} \varphi_{i}|\nabla v|^{2(s-1)} \varphi^{2 k-1} v^{1-\gamma} d x
\end{aligned}
$$

In Section 3 we will need a similar formula for the traceless Newton tensor:

Corollary 2.6. For any fixed $i$,

$$
\sum_{j} \partial_{j}\left(L_{i j}^{m}\right)=\frac{n-m}{n} \partial_{i} \sigma_{k}+n \sum_{j} L_{i j}^{m} v_{i} v^{-1}
$$

Proof. Follows easily from (2-5) and (2-2).

Lemma 2.7. If $\sigma_{1}, \ldots, \sigma_{m}>0$ and $m \leq n$, then

$$
\left\|T_{i j}^{m-1}\right\| \leq C_{m, n} \sigma_{m-1} .
$$

Proof. Because of Lemma 2.2, $T^{m-1}$ is positive definite. To estimate its norm we just need to look at its biggest eigenvalue. We are done, because

$$
\text { trace } T^{m-1}=(n-m) \sigma_{m-1} \text {. }
$$


Lemma 2.8. For any $1 \leq k \leq n-1$, if we have a metric $g=v^{-2}|d x|^{2}$ in the positive cone $\Gamma_{k}^{+}$, then

$$
\sum_{i, j} L_{i j}^{k} E_{i j} \geq 0
$$

with equality if and only if $E=0$.

Proof. Because $E_{i j}$ is traceless,

Using

$$
\sum_{i, j} L_{i j}^{k} E_{i j}=-\sum_{i, j} T_{i j}^{k} E_{i j}
$$

$$
E_{i j}=-\frac{1}{n} \sigma_{1} \delta_{i j}+A_{i j}, \quad(k+1) \sigma_{k+1}=T_{i j}^{k} A_{i j}, \quad T_{i j}^{k} \delta_{i j}=(n-k) \sigma_{k},
$$

we see that

$$
\sum_{i, j} T_{i j}^{k} E_{i j}=-\frac{n-k}{n} \sigma_{k} \sigma_{1}+(k+1) \sigma_{k+1}
$$

The result follows by the general inequality for matrices in the positive cone $\Gamma_{k}^{+}$:

$$
\sigma_{k+1} \leq \frac{n-k}{n(k+1)} \sigma_{1} \sigma_{k},
$$

with equality if and only if $E \equiv 0$.

\section{An Obata-type formula}

Obata's original result [1962] states that, if we have a metric $g$ on the unit sphere $\mathbb{S}^{n}$ that is conformal to the standard metric $g_{c}$ and of constant scalar curvature, then $E \equiv 0$; that is, $g$ is the standard metric $g_{c}$ or is obtained from it by a conformal diffeomorphism of the sphere. His method uses crucially the traceless Ricci tensor $E_{i j}=v v_{i j}-(1 / n) v \Delta v \delta_{i j}$ and the Bianchi identity $\nabla^{i} E_{i j}=\nabla^{j} R$. Indeed, his main step is to prove that

$$
\int_{\mathbb{S}^{n}} \sum_{i, j} E_{i j} E_{i j} v^{-1} d \operatorname{vol}_{g_{c}}=0,
$$

and thus establish that $g$ is an Einstein metric on $\mathbb{S}^{n}$.

This same argument was generalized for constant $\sigma_{k}$ (instead of constant $R$ ) by Viaclovsky [2000], with the role of $E$ played now by $L^{k}$ and the Bianchi identity replaced by (2-8). If the metric is defined on $\mathbb{R}^{n}$ instead of $\mathbb{S}^{n}$, an analogous argument works; however, a cutoff function $\eta$ is introduced and, in order to get the same conclusion, a careful estimate of the error terms is needed. We should also mention the work of Chang, Gursky, and Yang [2002; 2003] and of Li and Li [2002]. 
However, we are interested in the subcritical-problem approach of Gidas and Spruck [1981]; they have refined the computation of

$$
0 \leq \int_{B} \sum_{i, j} E_{i j} E_{i j} v^{-\delta} \eta d x=\cdots
$$

for any $\delta \in \mathbb{R}$. The main result of this section is the corresponding refinement for $\sigma_{k}$ :

Proposition 3.1. Let $\alpha>0$ and $n>2 k$. Take a solution $v$ of $\sigma_{k}(v)=v^{\alpha}$ in $U$, with $v \in \Gamma_{k}^{+}, v>0$, and $v^{-1} \in \mathscr{C}^{3}(U)$, where $U$ is a domain in $\mathbb{R}^{n}$. Pick $\eta \in \mathscr{C}_{0}^{\infty}(U)$ and a big positive integer $\theta$. There exist constants $d_{k-s}$ such that

$$
\begin{aligned}
\int_{U} \sum_{i, j} L_{i j}^{k} E_{i j} v^{-\delta} \eta^{\theta} & +\left(\frac{n-k}{n} \alpha-(1+n-\delta) \frac{k(n+2)}{2 n}\right) \int_{U} v^{\alpha}|\nabla v|^{2} v^{-\delta} \eta^{\theta} \\
& +(1+n-\delta) \sum_{s=1}^{k} d_{k-s} \int_{U} \sigma_{k-s}|\nabla v|^{2(s+1)} v^{-\delta} \eta^{\theta}=E_{1}(\eta)
\end{aligned}
$$

where

$$
E_{1}(\eta) \lesssim\left|\int_{U} \sum_{i, j} L_{i j}^{k} v_{i} \eta_{j} v^{1-\delta} \eta^{\theta-1}\right|+\left.\sum_{s=1}^{k}\left|\int_{U} \sum_{i, j} T_{i j}^{k-s} v_{j} \eta_{j}\right| \nabla v\right|^{2 s} v^{1-\delta} \eta^{\theta-1} \mid
$$

In addition, if $\delta$ is smaller than but close enough to $n+1$, all the coefficients in front of the integrals in (3-1) are positive.

Proof. One uses the inductive method developed in [González 2005b; 2005a] and the properties of $L^{k}$. In view of (2-4), integrate over $U$ to get

$$
\int \sum_{i, j} L_{i j}^{k} E_{i j} v^{-\delta} \eta^{\theta}=\int \sum_{i, j} L_{i j}^{k} v_{i j} v^{1-\delta} \eta^{\theta}-\frac{1}{n} \int \sum_{i, j} L_{i j}^{k}(\Delta v) v^{1-\delta} \delta_{i j} \eta^{\theta} .
$$

The last term vanishes since $L^{k}$ is trace-free. Integrating by parts and using (2-8),

$$
\begin{aligned}
& \int \sum_{i, j} L_{i j}^{k} E_{i j} v^{-\delta} \eta^{\theta} \\
& =-\int \sum_{i, j}\left(\partial_{i} L_{i j}^{k}\right) v_{j} v^{1-\delta} \eta^{\theta}-(1-\delta) \int \sum_{i, j} L_{i j}^{k} v_{i} v_{j} v^{-\delta} \eta^{\theta}-\int \sum_{i, j} L_{i j}^{k} v_{i} \eta_{j} v^{1-\delta} \eta^{\theta-1} \\
& =-\frac{n-k}{n} \int \sum_{i}\left(\partial_{i} \sigma_{k}\right) v_{i} v^{1-\delta} \eta^{\theta}-(1+n-\delta) \int \sum_{i, j} L_{i j}^{k} v_{i} v_{j} v^{-\delta} \eta^{\theta} \\
& -\int \sum_{i, j} L_{i j}^{k} v_{i} \eta_{j} v^{1-\delta} \eta^{\theta-1} .
\end{aligned}
$$


Group in $E_{1}(\eta)$ all the terms containing derivatives of $\eta$. Now compute, using $(2-1),(2-2)$, and (2-3):

$$
\begin{aligned}
\int \sum_{i, j} L_{i j}^{k} v_{i} v_{j} v^{-\delta} \eta^{\theta} & =\frac{n-k}{n} \int \sigma_{k}|\nabla v|^{2} v^{-\delta} \eta^{\theta}-\int \sum_{i, j} T_{i j}^{k} v_{i} v_{j} v^{-\delta} \eta^{\theta} \\
& =-\frac{k}{n} \int \sigma_{k}|\nabla v|^{2} v^{-\delta} \eta^{\theta}+\int \sum_{i, j, l} T_{i l}^{k-1} A_{l j} v_{i} v_{j} v^{-\delta} \eta^{\theta} \\
& =-\frac{k}{n} \int \sigma_{k}|\nabla v|^{2} v^{-\delta} \eta^{\theta}+\int \sum_{i, j, l} T_{i l}^{k-1} v_{l j} v_{i} v_{j} v^{1-\delta} \eta^{\theta} \\
& -\frac{1}{2} \int \sum_{i, j} T_{i j}^{k-1} v_{i} v_{j} v^{-\delta} \eta^{\theta}
\end{aligned}
$$

The middle term can be handled similarly to [González 2005b, Section 4]:

$$
\begin{aligned}
\int \sum_{i, j, l} T_{i l}^{k-1} v_{l j} v_{i} v_{j} v^{1-\delta} \eta^{\theta}=\frac{1}{2} \int \sum_{i, l} \partial_{l}\left(|\nabla v|^{2}\right) T_{i l}^{k-1} v_{i} v^{1-\delta} \eta^{\theta} \\
=-\frac{\delta-1}{2} \int \sum_{i, l} T_{i l}^{k-1} v_{i} v_{l}|\nabla v|^{2} v^{-\delta} \eta^{\theta}-\frac{1}{2} \int \sum_{i, l} \partial_{l}\left(T_{i l}^{k-1} v_{i}\right)|\nabla v|^{2} v^{1-\delta} \eta^{\theta} \\
-\frac{1}{2} \int \sum_{i, l} T_{i l}^{k-1} v_{i} \eta_{l}|\nabla v|^{2} v^{1-\delta} \eta^{\theta-1}
\end{aligned}
$$

To eliminate the term $\partial_{l}\left(T_{i l}^{k-1} v_{i}\right)$ from (3-4), just use the equality (2-5) and then substitute (3-4) into (3-3):

$$
\begin{aligned}
\int \sum_{i, j} L_{i j}^{k} v_{i} v_{j} v^{-\delta} \eta^{\theta} & \\
=-k \frac{n+2}{2 n} \int \sigma_{k}|\nabla v|^{2} v^{-\delta} \eta^{\theta} & -\frac{2+n-\delta}{2} \int \sum_{i, j} T_{i j}^{k-1} v_{i} v_{j}|\nabla v|^{2} v^{-\delta} \eta^{\theta} \\
& +\frac{n-k+1}{4} \int \sigma_{k-1}|\nabla v|^{4} v^{-\delta} \eta^{\theta}+E_{1}(\eta) \\
=-k \frac{n+2}{2 n} \int \sigma_{k}|\nabla v|^{2} v^{-\delta} \eta^{\theta} & +\mathscr{P}_{k-1}+E_{1}(\eta),
\end{aligned}
$$

where we have defined, for $k$ fixed and $s=1, \ldots, k-1$,

$$
\begin{aligned}
& \mathscr{B}_{k-s}= \\
& \quad-\frac{s+1+n-\delta}{s+1} \int \sum_{i, j} T_{i j}^{k-s} v_{i} v_{j}|\nabla v|^{2 s} v^{-\delta} \eta^{\theta}+\frac{n-k+s}{2(s+1)} \int \sigma_{k-s}|\nabla v|^{2(s+1)} v^{-\delta} \eta^{\theta} .
\end{aligned}
$$


The computations in (3-4) can be redone for $T^{k-s}$, and thus

$$
\mathscr{B}_{k-s}=\tilde{d}_{k-s} \int \sigma_{k-s}|\nabla v|^{2(s+1)} v^{-\delta} \eta^{\theta}+\tilde{c}_{k-s-1} \Re_{k-s-1}+E_{1}(\eta),
$$

with

$$
\tilde{d}_{k-s}=-\frac{s+n+1-\delta}{s+1}\left(1+\frac{k-s}{2(s+1)}\right)+\frac{n-k+s}{2(s+1)}, \quad \tilde{c}_{k-s}=\frac{(s+n+1-\delta)(s+2)}{2(s+1)^{2}} .
$$

The last step is

$$
\mathscr{B}_{1}=\tilde{d}_{1} \int \sigma_{1}|\nabla v|^{2 k} v^{-\delta} \eta+\tilde{c}_{1} \tilde{d}_{0} \int|\nabla v|^{2(k+1)} v^{-\delta} \eta .
$$

Substitute (3-6) into (3-5), inductively. This proves (3-1) for some constants $c_{k-s}$ and $d_{k-s}$ obtained from $\tilde{c}_{k-s}$ and $\tilde{d}_{k-s}$. Note that $c_{k-s}>0$ if $\delta<n+1$. We also want $d_{k-s}>0$ for $s=1, \ldots, k$, and this is achieved when $\delta$ is close enough to $n+1$ because $n>2 k$.

Lemma 3.2. With the same hypothesis as in the previous lemma,

$$
\begin{aligned}
\int_{U} v^{\alpha / k-\gamma} \eta^{\theta} & \lesssim\left(-1+\gamma-\frac{n}{2}\right) \int_{U} \sigma_{k}|\nabla v|^{2} v^{-\gamma} \eta^{\theta}+E_{2}(\eta), \text { where } \\
E_{2}(\eta) & \lesssim\left|\int_{U} \sum_{i} v_{i} \eta_{i} v^{1-\gamma} \eta^{\theta-1}\right| .
\end{aligned}
$$

Proof. Since $\sigma_{k}(v)=v^{\alpha}$ and $\sigma_{k} \leq C(n, k) \sigma_{1}^{k}$ (Lemma 2.2), we get $\sigma_{1}(v) \gtrsim v^{\alpha / k}$. It is easy to see that

$$
\int \sigma_{1} v^{-\gamma} \eta^{\theta}=\left(-1+\gamma-\frac{n}{2}\right) \int|\nabla v|^{2} v^{-\gamma} \eta^{\theta}+E_{2}(\eta),
$$

and the lemma is proved.

\section{Main estimates}

Here we obtain the needed $L^{p}$ estimate, as a consequence of (3-1). The terms on the left-hand side of (3-1) will be "good" terms, and we will give an estimate of the error terms.

Proposition 4.1. Take $n>2 k, \alpha \in(0, k)$, and let $v$ be a solution of (1-3). We have

(4-1) $\int_{\rho<|x|<M \rho} v^{\alpha(k+1) / k-\delta} \lesssim \frac{1}{\rho^{2(k+1)}} \int_{A_{\rho} \cup A_{M \rho}} v^{2(k+1)-\delta}+\frac{1}{\rho^{2}} \int_{A_{\rho} \cup A_{M \rho}} v^{2+\alpha-\delta}$,

for $\delta$ smaller than but close enough to $n+1$, and for $A_{\rho}=\left\{\frac{1}{2} \rho<|x|<\rho\right\}$ and $A_{M \rho}=\{M \rho<|x|<2 M \rho\}$; the constants depend on $M$ but not on $\rho$. 
Proof. If we take $\alpha-\delta=-\gamma$, then $-1-\frac{1}{2} n+\gamma>0$, and the preceding lemma allows us, in (3-1), to replace

$$
\int|\nabla v|^{2} v^{\alpha-\delta} \eta^{\theta} \quad \text { by } \quad \int v^{\alpha(k+1) / k-\delta} \eta^{\theta}+E_{2}(\eta) .
$$

Let $\eta$ be a smooth cutoff function such that

$$
\eta= \begin{cases}1 & \text { if } \rho<|x|<M \rho, \\ 0 & \text { if } 0<|x|<\frac{1}{2} \rho \text { and } 2 M \rho<|x|,\end{cases}
$$

$|\nabla \eta| \lesssim 1 / \rho$, and $\left|D^{2} \eta\right| \lesssim 1 / \rho^{2}$. The error $E_{1}(\eta)$ in (3-2) is of one of these two types:

$$
\begin{aligned}
& E_{11}(\eta) \lesssim\left|\int_{A_{\rho} \cup A_{M_{\rho}}} \sum_{i, j} L_{i j}^{k} v_{i} \eta_{j} v^{1-\delta} \eta^{\theta-1}\right|, \quad \text { or } \\
& \left.E_{12}(\eta) \lesssim \sum_{s=1}^{k}\left|\int_{A_{\rho} \cup A_{M \rho}} \sum_{i, j} T_{i j}^{k-s} v_{j} \eta_{j}\right| \nabla v\right|^{2 s} v^{1-\delta} \eta^{\theta-1} \mid .
\end{aligned}
$$

These will be handled as in the proof of [González 2005a, Theorem 1.1], but here we present a clearer proof for this particular cutoff.

To understand $E_{11}$, substitute $L^{k}=(1-k / n) \sigma_{k} I-T^{k}$, so that

$$
E_{11}(\eta) \lesssim \int_{A_{\rho} \cup A_{M \rho}} \sigma_{k} v_{i} \eta_{i} v^{1-\delta} \eta^{\theta-1}+\int_{A_{\rho} \cup A_{M \rho}} T_{i j}^{k} v_{i} \eta_{j} v^{1-\delta} \eta^{\theta-1}
$$

We cannot use the standard trick — to estimate the norm $\left\|T^{k}\right\| \lesssim \sigma_{k}$ as in Lemma 2.7 - because we cannot conclude that $T^{k}$ is positive definite from the information on $\sigma_{1}, \ldots, \sigma_{k}$, and we need to write everything in terms of smaller $T^{k-s}$ 's. An inductive process is needed.

Substitute $T_{i j}^{k}=\sigma_{k} \delta_{i j}-A_{i l} T_{l j}^{k-1}$ and $A_{i l}=v v_{i l}-\frac{1}{2}|\nabla v|^{2} \delta_{i l}$ in (4-2). Together with Lemma 2.7, we have

$$
\begin{aligned}
E_{11}(\eta) \lesssim \int \sigma_{k}|\nabla v||\nabla \eta| v^{1-\delta} \eta^{\theta-1}+\int \sigma_{k-1}|\nabla v|^{3}|\nabla \eta| v^{1-\delta} \eta^{\theta-1} \\
+\left|\int T_{l j}^{k-1} v_{i l} v_{i} \eta_{j} v^{2-\delta} \eta^{\theta-1}\right|
\end{aligned}
$$

For the last term, proceed as in (3-4):

$$
\begin{array}{r}
\int T_{l j}^{k-1} v_{i l} v_{i} \eta_{j} v^{2-\delta} \eta^{\theta-1}=\frac{1}{2} \int \partial_{l}\left(|\nabla v|^{2}\right) T_{l j}^{k-1} \eta_{j} v^{2-\delta} \eta^{\theta-1} \\
=-\frac{1}{2} \int\left(\partial_{l} T_{l j}^{k-1}\right)|\nabla v|^{2} \eta_{j} v^{2-\delta} \eta^{\theta-1}-\frac{1}{2} \int T_{l j}^{k-1}|\nabla v|^{2} \eta_{l j} v^{2-\delta} \eta^{\theta-2} \\
-\frac{2-\delta}{2} \int T_{l j}^{k-1} \eta_{l} v_{j}|\nabla v|^{2} v^{1-\delta} \eta^{\theta-1}
\end{array}
$$


Note that (2-5) helps to compute $\partial_{l} T_{l j}^{k-1}$, and thus, from (4-4) and Lemma 2.7,

$$
\begin{aligned}
\left|\int T_{l j}^{k-1} v_{i l} v_{i} \eta_{j} v^{2-\delta} \eta^{\theta-1}\right| \\
\quad \lesssim \int \sigma_{k-1}\left|D^{2} \eta\right||\nabla v|^{2} v^{2-\delta} \eta^{\theta-2}+\int \sigma_{k-1}|\nabla v|^{3}|\nabla \eta| v^{1-\delta} \eta^{\theta-1}
\end{aligned}
$$

Young's inequality for a small $\varepsilon$, together with (4-3) and (4-5), gives

$$
\begin{aligned}
E_{11}(\eta) \lesssim \varepsilon \int \sigma_{k}|\nabla v|^{2} \eta^{\theta} v^{-\delta}+\frac{C_{\varepsilon}}{\rho^{2}} \int_{A_{\rho} \cup A_{M \rho}} \sigma_{k} v^{2-\delta} \eta^{\theta-2} \\
+\varepsilon \int \sigma_{k-1}|\nabla v|^{4} \eta^{\theta} v^{-\delta}+\frac{C_{\varepsilon}}{\rho^{4}} \int_{A_{\rho} \cup A_{M \rho}} \sigma_{k-1} v^{4-\delta} \eta^{\theta-4}
\end{aligned}
$$

To finish the estimate, we just need (4-7) from the next lemma, applied iteratively:

$$
E_{11}(\eta) \lesssim \varepsilon \sum_{s=0}^{k} \int \sigma_{k-s}|\nabla v|^{2(s+1)} \eta^{\theta} v^{-\delta}+\frac{C_{\varepsilon}}{\rho^{2(k+1)}} \int_{A_{\rho} \cup A_{M \rho}} v^{2(k+1)-\delta}
$$

The estimate for $E_{12}(\eta)$ follows in a similar manner. For the error in $E_{2}(\eta)$, defined in 3-7, we use Young's inequality with $p=q=2$ :

$$
E_{2}(\eta) \lesssim \int|\nabla v||\nabla \eta| v^{1-\gamma} \eta^{\theta-1} \lesssim \varepsilon \int|\nabla v|^{2} v^{\alpha-\delta} \eta^{\theta}+\frac{C_{\varepsilon}}{\rho^{2}} \int_{A_{\rho} \cup A_{M \rho}} v^{2+\alpha-\delta}
$$

Putting it all together in (3-1), and taking into account that $\sum_{i, j} L_{i j}^{k} E_{i j} \geq 0$,

$$
\begin{aligned}
\int_{\rho<|x|<M \rho} v^{\alpha(k+1) / k-\delta} & \leq \int v^{\alpha(k+1) / k-\delta} \eta^{\theta} \\
& \lesssim \frac{1}{\rho^{2(k+1)}} \int_{A_{\rho} \cup A_{M \rho}} v^{2(k+1)-\delta}+\frac{1}{\rho^{2}} \int_{A_{\rho} \cup A_{M \rho}} v^{2+\alpha-\delta}
\end{aligned}
$$

Lemma 4.2. For all $\varepsilon>0$ and $s=0, \ldots, k-1$, and for $\theta$ a big positive integer,

$$
\begin{aligned}
& \frac{1}{\rho^{2(s+1)}} \int \sigma_{k-s} v^{2(s+1)-\delta} \eta^{\theta-2(s+1)} \\
& \leq \varepsilon \int \sigma_{k-s-1}|\nabla v|^{2(s+2)} \eta^{\theta} v^{-\delta}+\frac{C_{\varepsilon}}{\rho^{2(s+2)}} \int_{\{|\nabla \eta| \neq 0\}} \sigma_{k-s-1} \eta^{\theta-2(s+2)} v^{2(s+2)-\delta} .
\end{aligned}
$$


Proof. First use the "divergence" formula (2-6) for $\sigma_{k-s}$ with integration by parts:

$$
\begin{aligned}
& (k-s) \int \sigma_{k-s} v^{2(s+1)-\delta} \eta^{\theta-2(s+1)} \\
& =\frac{n-k+s+1}{2} \int \sigma_{k-s-1}|\nabla v|^{2} \eta^{\theta-2(s+1)} v^{2(s+1)-\delta} \\
& \quad-(n+2(s+1)-\delta+1) \int T_{i j}^{k-s-1} v_{i} v_{j} \eta^{\theta-2(s+1)} v^{2(s+1)-\delta} \\
& \quad-\int T_{i j}^{k-s-1} v_{i} \eta_{j} \eta^{\theta-2(s+1)-1} v^{2(s+1)-\delta+1} .
\end{aligned}
$$

Use Lemma 2.7 again to bound the norm of the Newton tensor in (4-8):

$$
\begin{aligned}
\int \sigma_{k-s} v^{2(s+1)-\delta} \eta^{\theta-2(s+1) \lesssim} & \int \sigma_{k-s-1}|\nabla v|^{2} \eta^{\theta-2(s+1)} v^{2(s+1)-\delta} \\
& +\frac{1}{\rho} \int \sigma_{k-s-1}|\nabla v| \eta^{\theta-2(s+1)-1} v^{2(s+1)-\delta+1} .
\end{aligned}
$$

Young's inequality with $\varepsilon$ and $p=s+2, q=(s+2) /(s+1)$ now reads

$$
\begin{aligned}
& \int \sigma_{k-s-1}|\nabla v|^{2} \eta^{\theta-2(s+1)} v^{2(s+1)-\delta} \\
& \lesssim \varepsilon \rho^{2(s+1)} \int \sigma_{k-s-1}|\nabla v|^{2(s+1)} \eta^{\theta} v^{-\delta}+\frac{C_{\varepsilon}}{\rho^{2}} \int \sigma_{k-s-1} \eta^{\theta-2(s+2)} v^{2(s+2)-\delta} .
\end{aligned}
$$

For the second part in (4-9), take $p=2(s+2)$ and $q=\frac{2(s+2)}{2(s+2)-1}$ :

$$
\begin{aligned}
& \frac{1}{\rho} \int \sigma_{k-s-1}|\nabla v| \eta^{\theta-2(s+2)-1} v^{2(s+1)-\delta+1} \\
& \lesssim \varepsilon \rho^{2(s+1)} \int \sigma_{k-s-1}|\nabla v|^{2(s+2)} \eta^{\theta} v^{-\delta}+\frac{C_{\varepsilon}}{\rho^{2}} \int \sigma_{k-s-1} \eta^{\theta-2(s+2)} v^{2(s+2)-\delta}
\end{aligned}
$$

The lemma is proved by substituting (4-10) and (4-11) into (4-9).

Proposition 4.3. For $n \geq 2(k+1), \alpha \in(0, k)$, and $v$ a solution of (1-3), we have

$$
\int_{\rho<|x|<M \rho} v^{\alpha(k+1) / k-\delta} \leq C \rho^{n-(\delta-\alpha(k+1) / k) /(1-\alpha / 2 k)},
$$

where $C$ depends on $M$ and $\delta$, but not on $\rho$.

Proof. Use Hölder's inequality with

to get

$$
p=\frac{\delta-\alpha(k+1) / k}{\delta-2(k+1)} \quad \text { and } \quad q=\frac{p}{p-1}
$$

$$
\frac{1}{\rho^{2(k+1)}} \int_{A_{\rho} \cup A_{M \rho}} v^{2(k+1)-\delta} \leq \varepsilon \int_{A_{\rho} \cup A_{M \rho}} v^{\alpha(k+1) / k-\delta}+C_{\varepsilon} \rho^{n-2(k+1) q},
$$


for some small $\varepsilon$, to be chosen later. Also, a Hölder estimate with

gives

$$
\tilde{p}=\frac{\delta-\alpha(k+1) / k}{\delta-2-\alpha} \quad \text { and } \quad \tilde{q}=\frac{\tilde{p}}{\tilde{p}-1}
$$

$$
\frac{1}{\rho^{2}} \int_{A_{\rho} \cup A_{M \rho}} v^{2+\alpha-\delta} \leq \varepsilon \int_{A_{\rho} \cup A_{M \rho}} v^{\alpha(k+1) / k-\delta}+C_{\varepsilon} \rho^{n-2 \tilde{q}} .
$$

When $\alpha \in(0, k)$ and $\delta$ is close enough to $n+1$, then $p, \tilde{p}>1$. Look at the powers of $\rho$ in (4-13) and (4-14):

$$
n-2(k+1) q=n-2 \tilde{q}=n-\frac{\delta-\alpha(k+1) / k}{1-\alpha / 2 k} .
$$

Choosing $\varepsilon$ small enough, we conclude from (4-1) that

$$
\int_{\rho<|x|<M \rho} v^{\alpha(k+1) / k-\delta} \leq C \rho^{n-(\delta-\alpha(k+1) / k) /(1-\alpha / 2 k)} .
$$

\section{Proof of Theorem 1.1}

The next proposition is similar to the study of the critical problem in [González 2005a]. In particular, a volume finiteness condition gives regularity near the singularity.

Proposition 5.1. Take $\alpha \in(0, k)$ and $n>2 k$, and let $v$ be a solution of (1-3) on $B_{\rho}\left(x_{0}\right) \subset B$, with $v>0$ and $v \in \Gamma_{k}^{+}$. If

$$
\int_{B_{\rho}\left(x_{0}\right)} v^{(\alpha-2 k) n /(2 k)} \leq a
$$

for some small enough a (not depending on $\rho$ ), then

$$
\sup _{B_{\rho / 2}\left(x_{0}\right)}\left|v^{-1}\right| \leq \frac{C}{\rho^{n / p}}\left\|v^{-1}\right\|_{L^{p}\left(B_{\rho}\left(x_{0}\right)\right)}
$$

for all $p>(n-2 k) k /(k+1)$. In particular, if

$$
\int_{\varepsilon<|x|<1} v^{(\alpha-2 k) n /(2 k)}<C<\infty
$$

for some constant $C$ independent of $\varepsilon$, the function $v$ is bounded near the origin.

Proof. The argument is similar to [González 2005a, Theorem 1.2] for the critical problem. Condition (5-2) is analogous to its volume smallness condition.

Proof of Theorem 1.1. Fix $x_{0}$ small enough and take $2 R=\left|x_{0}\right|$. First, note that Hölder estimates with

$$
r=\frac{\delta-(k+1) / k}{(2 k-\alpha) n /(2 k)}>1 \quad \text { and } \quad 1=\frac{1}{r}+\frac{1}{s}
$$


give, independently of $x_{0}$,

$$
\begin{aligned}
\int_{B_{R}\left(x_{0}\right)} v^{(\alpha-2 k) n /(2 k)} & \leq\left(\int_{R \leq|x| \leq 3 R} v^{(k+1) / k-\delta}\right)^{1 / r} \varepsilon^{n / s} \\
& \lesssim R^{\left(n-\frac{\delta-\alpha(k+1) / k}{1-\alpha /(2 k)}\right) \frac{1}{r} \frac{n}{s}} \lesssim R^{0}<\infty
\end{aligned}
$$

We cannot apply Proposition 5.1 directly to $v$. However, we could have started with the function $\tilde{v}(y)=A^{2 k /(2 k-\alpha)} v(y / A)$ that still satisfies the same equation $\sigma_{k}(\tilde{v})=\tilde{v}^{\alpha}$, for some $A$ big enough and of the form

$$
A=(\text { constant }) \int_{R \leq|x| \leq 3 R} v^{(\alpha-2 k) n /(2 k)} .
$$

Since we are interested only in the local behavior near zero, we can assume that (5-1) gives an estimate for $v$,

$$
\sup _{B_{R / 2}\left(x_{0}\right)}\left|v^{-1}\right| \leq \frac{C}{R^{n / p}}\left\|v^{-1}\right\|_{L^{p}\left(B_{R}\left(x_{0}\right)\right)}
$$

for all $p>(n-2 k) k /(k+1)$, and with $C$ depending on

$$
\int_{R \leq|x| \leq 3 R} v^{(\alpha-2 k) n /(2 k)}
$$

This estimate is uniformly bounded by a constant, independently of $R$, because of (5-3). It is also true that

$$
\sup _{B_{R / 2}\left(x_{0}\right)}\left|v^{-1}\right| \leq \frac{C}{\left|x_{0}\right|^{n / p}}\left\|v^{-1}\right\|_{L^{p}(\{R \leq|x| \leq 3 R\})}
$$

for all $p>(n-2 k) k /(k+1)$. Set $p=\delta-\alpha(k+1) / k$; this choice is valid when $\alpha \in(0, k)$ and $n>2 k$. Use (4-12) again:

$$
\int_{R \leq|x| \leq 3 R} v^{-p} \leq C\left|x_{0}\right|^{n-\frac{p}{1-\alpha /(2 k)}}
$$

and thus, from (5-4), we arrive at

as desired.

$$
v^{-1}\left(x_{0}\right) \leq \frac{C}{\left|x_{0}\right|^{2 k /(2 k-\alpha)}}
$$

Corollary 5.2 (Harnack). Under these hypotheses, there exists $M_{0}>0$ such that, for all $\rho>0$ and $M \leq M_{0}$,

$$
\sup _{\rho \leq|x| \leq \rho M} v^{-1} \leq C \inf _{\rho \leq|x| \leq \rho M} v^{-1},
$$

where $C$ is independent of $v, \rho$, and $M$. 
Proof. Once we get a supremum estimate (5-4) for a ball, standard elliptic theory yields the infimum estimate. If we write $v^{-2}=u^{2 /(n-2)}$, then $u$ is a superharmonic function. To finish, use a covering argument for the annulus $\{\rho \leq|x| \leq \rho M\}$.

Corollary 5.3. If $v$ is a solution of (1-3), then either $v^{-1}$ is bounded near the origin, or $v^{-1}(x) \rightarrow \infty$ as $x \rightarrow 0$.

Proof. The argument follows the steps of [Gidas and Spruck 1981, Corollary 3.3], by using the second part of Proposition 5.1.

\section{Proof of Theorem 1.2}

We have proved the estimate

$$
v^{-1}(x) \leq \frac{C}{|x|^{2 k /(2 k-\alpha)}}
$$

Now we would like to get the opposite inequality. Suppose that

$$
\lim _{x \rightarrow 0} \inf |x|^{2 k /(2 k-\alpha)} v^{-1}(x)=0 .
$$

From the Harnack estimate (5-5) follows that

$$
\lim _{x \rightarrow 0}|x|^{2 k /(2 k-\alpha)} v^{-1}(x)=0 .
$$

We want to see that in this case the function $v^{-1}$ is bounded near the origin and thus that the theorem follows. It suffices to establish (5-2).

Let's review two results from [González 2005a]:

Proposition 6.1. Let $v$ be a solution with $v^{-1} \in \mathscr{C}^{3}(U), v>0, v \in \Gamma_{k}^{+}$, and $n>2 k$. For all $\varphi \in \mathscr{C}_{0}^{\infty}(U)$ and $\theta$ a big positive integer,

$$
\int_{U} \sigma_{k} \varphi^{\theta} v^{-\gamma} \geq \sum_{s=1}^{k} c_{k-s}(\gamma) \int_{U} \sigma_{k-s}|\nabla v|^{2 s} \varphi^{\theta} v^{-\gamma}+E(\varphi),
$$

where

$$
\left.E(\varphi) \lesssim \sum_{s=1}^{k}\left|\int_{U} \sum_{i, j} T_{i j}^{k-s} v_{j} \varphi_{i}\right| \nabla v\right|^{2(s-1)} \varphi^{\theta-1} v^{1-\gamma} \mid,
$$

and where the coefficients $c_{k-s}(\gamma)$ are positive for all $\gamma$ with

$$
\gamma>n-\frac{n-2 k}{k+1}
$$

Proposition 6.2. For all $\varepsilon>0$, the error term (6-4) can be estimated by

$$
E(\varphi) \leq \varepsilon \sum_{s=1}^{k} \int \sigma_{k-s}|\nabla v|^{2 s} \varphi^{\theta} v^{-\gamma}+C_{\varepsilon} \sum_{U_{k}} \int U_{k}(\varphi) \varphi^{\theta-\alpha_{k}} v^{2 k-\gamma},
$$


where the $U_{k}(\varphi)$ 's are groups of derivatives of $\varphi$ of order $2 k$, and $\alpha_{k} \in \mathbb{R}$ are constants depending on each of the $U_{k}$ 's. These concepts are defined inductively in the following manner:

- For a fixed $s=1, \ldots, k$, the starting point is

$$
U_{s}(\varphi) \varphi^{\alpha_{s}}=|\nabla \varphi|^{2 s} \varphi^{-2 s} .
$$

- For each integer $l=0,1, \ldots$ and $m=s+l$, and once given $U_{m} \varphi^{\alpha_{m}}$, the following step is of one of these three shapes:

$$
U_{m+1} \varphi^{-\alpha_{m+1}}=\left\{\begin{array}{l}
U_{m}^{(m+1) / m} \varphi^{-\alpha_{m}(m+1) / m} \\
\left|\nabla U_{m}\right|^{\frac{2(m+1)}{2(m+1)-1}} \varphi^{-\alpha_{m} \frac{2(m+1)}{2(m+1)-1}} \\
\left(|\nabla \varphi|^{2} U_{m}\right) \varphi^{-\alpha_{m}-2} .
\end{array}\right.
$$

- The ending point is when $m=s+l$ reaches $k$.

We will use (6-3) for a suitable cutoff function. Take $\varphi=\eta r$ with $\eta \in \mathscr{C}_{0}^{\infty}(B \backslash\{0\})$, such that

$$
\eta= \begin{cases}1 & \text { if } \varepsilon<|x|<R, \\ 0 & \text { if }|x|<\frac{1}{2} \varepsilon \text { and }|x|>2 R,\end{cases}
$$

and so that the derivatives have a good bound on $\frac{1}{2} \varepsilon<|x|<\varepsilon$ and $R<|x|<2 R$. The value of $\gamma$ will be chosen later. Rewrite (6-3) as

$$
\int \sigma_{k} v^{-\gamma} \varphi^{\theta} \gtrsim \sum_{s=1}^{k} \int \sigma_{k-s}|\nabla v|^{2 s} v^{-\gamma} \varphi^{\theta}-\int T_{i j}^{k-1} v_{i} \varphi_{j} \varphi^{\theta-1} v^{1-\gamma}+\tilde{E}(\varphi),
$$

with

$$
\left.\tilde{E}(\varphi) \lesssim \sum_{s=2}^{k}\left|\int T_{i j}^{k-s} v_{i} \varphi_{j}\right| \nabla v\right|^{2(s-1)} \varphi^{\theta-1} v^{1-\gamma} \mid,
$$

since we will look more carefully at the term in $T^{k-1}$. Integration by parts gives

$$
\begin{aligned}
-\int \sum_{i, j} T_{i j}^{k-1} v_{i} \varphi_{j} \varphi^{\theta-1} v^{1-\gamma}=-\frac{1}{2-\gamma} \int \sum_{i, j} T_{i j}^{k-1} \partial_{i}\left(v^{2-\gamma}\right) \varphi_{j} \varphi^{\theta-1} \\
=\frac{1}{2-\gamma} \int \sum_{i, j} T_{i j}^{k-1} \varphi_{i j} \varphi^{\theta-1} v^{2-\gamma}+\frac{1}{2-\gamma} \int \sum_{i, j} \partial_{i}\left(T_{i j}^{k-1}\right) \varphi_{j} \varphi^{\theta-1} v^{2-\gamma} \\
+\frac{\theta-1}{2-\gamma} \int \sum_{i, j} T_{i j}^{k-1} \varphi_{i} \varphi_{j} \varphi^{\theta-2} v^{2-\gamma}
\end{aligned}
$$


Substituting (2-5) into this, we get

$$
\begin{aligned}
& -(n+2-\gamma) \int \sum_{i, j} T_{i j}^{k-1} v_{i} \varphi_{j} \varphi^{\theta-1} v^{1-\gamma}=\int \sum_{i, j} T_{i j}^{k-1} \varphi_{i j} \varphi^{\theta-1} v^{2-\gamma} \\
& -(n-k+1) \int \sum_{i} \sigma_{k-1} v_{i} \varphi_{i} \varphi^{\theta-1} v^{1-\gamma}+(\theta-1) \int \sum_{i, j} T_{i j}^{k-1} \varphi_{i} \varphi_{j} \varphi^{\theta-2} v^{2-\gamma} .
\end{aligned}
$$

Now substitute this into (6-7):

$$
\begin{array}{r}
\int \sigma_{k} \varphi^{\theta} v^{-\gamma} \gtrsim \sum_{s=1}^{k} \int \sigma_{k-s}|\nabla v|^{2 s} \varphi^{\theta} v^{-\gamma}+\frac{1}{n+2-\gamma} \int \sum_{i, j} T_{i j}^{k-1} \varphi_{i j} \varphi^{\theta-2} v^{2-\gamma} \\
+\frac{\theta-1}{n+2-\gamma} \int \sum_{i, j} T_{i j}^{k-1} \varphi_{i} \varphi_{j} \varphi^{\theta-2} v^{2-\gamma} \\
\quad-\frac{n-k+1}{n+2-\gamma} \int \sum_{i} \sigma_{k-1} v_{i} \varphi_{i} \varphi^{\theta-1} v^{1-\gamma}+\tilde{E}(\varphi) .
\end{array}
$$

Group all the error terms into

$$
E(\varphi) \lesssim \sum_{s=1}^{k} \int \sigma_{k-s}|\nabla \varphi||\nabla v| \varphi^{\theta-1} v^{1-\gamma}
$$

Compute

$$
\begin{aligned}
\varphi_{i} & =\frac{x_{i}}{r} \eta+E_{1}(\varphi), \\
\varphi_{i j} & =r^{-1}\left(-\frac{x_{i} x_{j}}{r^{2}}+\delta_{i j}\right) \eta+E_{1}(\varphi), \\
\sum_{i, j} T_{i j}^{k-1} \varphi_{i j} & =r^{-1}\left(-\sum_{i, j} T_{i j}^{k-1} \frac{x_{i} x_{j}}{r^{2}}+(n-k+1) \sigma_{k-1}\right) \eta+E_{1}(\varphi) .
\end{aligned}
$$

Since $T^{k-1}$ is positive definite and trace $T^{k-1}=(n-k+1) \sigma_{k-1}$, as long as we keep $1<\theta$ we have

$$
\sum_{i, j} T_{i j}^{k-1}\left(\varphi_{i j}+(\theta-1) \varphi_{i} \varphi_{j} r^{-\theta}\right) \geq C(\theta) \sigma_{k-1} r^{-1} \eta^{2}+E_{1}(\varphi)
$$

for some $C(\theta)>0$. If we keep $\gamma<n+2$, we can conclude from (6-8) that

$$
\begin{aligned}
E(\varphi)+E_{1}(\varphi)+\int \sigma_{k} \varphi^{\theta} v^{-\gamma} & \\
& \gtrsim \sum_{s=1}^{k} \int \sigma_{k-s}|\nabla v|^{2 s} \varphi^{\theta} v^{-\gamma}+\int \sigma_{k-1} r^{-2} \varphi^{\theta} v^{2-\gamma}
\end{aligned}
$$


We have not been very precise with the errors $E_{1}(\varphi)$; however, they are of a similar type to $E(\varphi)$ and can be treated in the same manner. Note that, in the positive cone,

$$
\sigma_{k-1} \gtrsim \sigma_{k}^{(k-1) / k}=v^{\alpha(k-1) / k},
$$

so, with (6-9) we have actually proved

(6-10) $\quad E(\varphi) \gtrsim \int\left(v^{\alpha(k-1) / k+2-\gamma} r^{-2}-v^{\alpha-\gamma}\right) \varphi^{\theta}+\sum_{s=1}^{k} \int \sigma_{k-s}|\nabla v|^{2 s} \varphi^{\theta} v^{-\gamma}$.

To handle $E(\varphi)$, we need to control the error terms that appear in Proposition 6.2.

Using Lemma 6.3 below,

$$
\begin{aligned}
& \int U_{k}(\varphi) \varphi^{\theta-\alpha_{k}} v^{2 k-\gamma} \\
& \quad \lesssim \int r^{-2 k} \varphi^{\theta} v^{2 k-\gamma}+\frac{1}{\varepsilon^{2 k}} \int_{\varepsilon / 2<|x|<\varepsilon} r^{\theta} v^{2 k-\gamma}+\frac{1}{R^{2 k}} \int_{R<|x|<2 R} r^{\theta} v^{2 k-\gamma}
\end{aligned}
$$

Looking one-by-one at the terms above, notice that

$$
\frac{1}{\varepsilon^{2 k}} \int_{\varepsilon / 2<|x|<\varepsilon} r^{\theta} v^{2 k-\gamma} \rightarrow 0 \quad \text { as } \varepsilon \rightarrow 0,
$$

by using the previous estimate (6-1) and the definition of $\eta$, and as soon as

$$
\gamma>n-\alpha\left(\frac{n-2 k}{2 k}\right) \text {. }
$$

A similar argument gives

$$
\frac{1}{R^{2 k}} \int_{R<|x|<2 R} r^{\theta} v^{2 k-\gamma} \leq C .
$$

The other integral in (6-11) is bounded by

$$
\int r^{-2 k} \varphi^{\theta} v^{2 k-\gamma} \lesssim \int\left(v^{\alpha(k-1) / k+2-\gamma} r^{-2}\right)\left(v^{-\alpha(k-1) / k-2+2 k} r^{2-2 k}\right) \varphi^{\theta} .
$$

Our assumption (6-2) yields

$$
v^{-\alpha(k-1) / k-2+2 k} r^{2-2 k}=o(1),
$$

and thus from (6-10) we obtain

$$
C \gtrsim \int\left(v^{\alpha(k-1) / k+2-\gamma} r^{-2}-v^{\alpha-\gamma}\right) \varphi^{\theta} .
$$

Again, because of (6-2), we have

Theorem 1.1 gives

$$
r^{2} v^{\alpha / 2-k}=o(1) .
$$




$$
v^{\alpha-\gamma} \lesssim\left(v^{\alpha(k-1) / k+2-\gamma} r^{-2}\right)\left(r^{2} v^{(\alpha-2 k) / 2}\right) .
$$

Comparing the orders, we quickly obtain

$$
\int v^{\alpha(k-1) / k} r^{-2} v^{2-\gamma} \varphi^{\theta}<\infty .
$$

This is precisely the term (5-2) that we need to estimate, because

$$
\begin{aligned}
\int_{\varepsilon \leq|x| \leq R} v^{(\alpha-2 k) n /(2 k)} & =\int v^{\alpha(k-1) / k+2-\gamma} v^{-\alpha(k-1) / k-n+\alpha n /(2 k)-2+\gamma} \eta^{\theta} \\
& \lesssim \int v^{\alpha(k-1) / k+2-\gamma} r^{\left(-\alpha \frac{k-1}{k}-n+\alpha \frac{n}{2 k}-2+\gamma\right)\left(\frac{2 k}{2 k-\alpha}\right)} \eta^{\theta} \\
& =\int v^{\alpha(k-1) / k+2-\gamma} r^{-2} \varphi^{\theta}
\end{aligned}
$$

after using Theorem 1.1 and choosing $\theta$ and $\gamma$ so that

$$
\left(-\alpha \frac{k-1}{k}-n+\alpha \frac{n}{2 k}-2+\gamma\right)\left(\frac{2 k}{2 k-\alpha}\right)=-2+\theta,
$$

that is, picking

$$
\gamma=n-\alpha\left(\frac{n-2 k}{2 k}\right)+\theta\left(1-\frac{\alpha}{2 k}\right)
$$

This is an admissible value for $\gamma$ because, when $\alpha<2 k /(k+1)$, it can be chosen to satisfy (6-5), (6-12), $\gamma<n+2$, and $\theta>1$.

Lemma 6.3. For the cutoff $\varphi=r \eta$ constructed in the previous proof,

$$
U_{k}(\varphi) \varphi^{\theta-\alpha_{k}} \lesssim r^{-2 k} \varphi^{\theta}+\varepsilon^{-2 k} r^{\theta} \chi_{\{\varepsilon / 2 \leq|x|<\varepsilon\}}+R^{-2 k} r^{\theta} \chi_{\{R \leq|x|<2 R\}}
$$

Proof. The definition of the $U_{k}$ was given in Proposition 6.2. We are just interested in the orders of $r$ and $\varepsilon$. For fixed $s=1, \ldots, k$, the initial step is

$$
\begin{aligned}
U_{s}(\varphi) \varphi^{\theta-2 s}=|\nabla \varphi|^{2 s} \varphi^{\theta-2 s} & \\
& \lesssim|\nabla r|^{2 s} \varphi^{\theta-2 s} \eta^{2 s}+|\nabla \eta|^{2 s} r^{2 s} \varphi^{\theta-2 s} \lesssim r^{-2 s} \varphi^{\theta}+\varepsilon^{-2 s} r^{\theta} \eta^{2 s} .
\end{aligned}
$$

Next, assume that the result is true for $m=s+l$ :

$$
U_{m}(\varphi) \varphi^{\theta-\alpha_{m}} \lesssim r^{-2 m} \varphi^{\theta}+\varepsilon^{-2 m} r^{\theta} \eta^{2 m} .
$$

The proof for $m+1$ follows easily from (6-6).

\section{References}

[Caffarelli, Gidas, and Spruck 1989] L. A. Caffarelli, B. Gidas, and J. Spruck, "Asymptotic symmetry and local behavior of semilinear elliptic equations with critical Sobolev growth", Comm. Pure Appl. Math. 42:3 (1989), 271-297. MR 90c:35075 Zbl 0702.35085 
[Chang, Gursky, and Yang 2002] S.-Y. A. Chang, M. J. Gursky, and P. Yang, "An a priori estimate for a fully nonlinear equation on four-manifolds", J. Anal. Math. 87 (2002), 151-186. MR 2003k:53036 Zbl 1067.58028

[Chang, Gursky, and Yang 2003] S.-Y. A. Chang, M. J. Gursky, and P. C. Yang, "Entire solutions of a fully nonlinear equation", pp. 43-60 in Lectures on partial differential equations, edited by S.-Y. A. Chang et al., New Stud. Adv. Math. 2, International Press, Somerville, MA, 2003. MR 2005b:53053 Zbl 02072579

[Gårding 1959] L. Gårding, "An inequality for hyperbolic polynomials”, J. Math. Mech. 8 (1959), 957-965. MR 22 \#4809 Zbl 0090.01603

[Gidas and Spruck 1981] B. Gidas and J. Spruck, "Global and local behavior of positive solutions of nonlinear elliptic equations”, Comm. Pure Appl. Math. 34:4 (1981), 525-598. MR 83f:35045 Zbl 0465.35003

[González 2005a] M. d. M. González, "Removability of singularities for a class of fully non-linear elliptic equations", preprint, 2005, Available at http://www.ma.utexas.edu/users/mgonzale/Papers/ Removability_singularities.p\% df.

[González 2005b] M. d. M. González, "Singular sets of a class of locally conformally flat manifolds”, Duke Math. J. 129:3 (2005), 551-572. MR 2006d:53034 Zbl 05010589

[Gursky and Viaclovsky 2005] M. Gursky and J. Viaclovsky, "Convexity and singularities of curvature equations in conformal geometry", preprint, 2005. math.DG/0504066

[Han 2004] Z.-C. Han, "Local pointwise estimates for solutions of the $\sigma_{2}$ curvature equation on 4-manifolds", Int. Math. Res. Not. 79 (2004), 4269-4292. MR 2126828 Zbl 02151806

[Lee and Parker 1987] J. M. Lee and T. H. Parker, "The Yamabe problem", Bull. Amer. Math. Soc. (N.S.) 17:1 (1987), 37-91. MR 88f:53001 Zbl 0633.53062

[Li 2006] Y. Y. Li, "Conformally invariant fully nonlinear elliptic equations and isolated singularities”, J. Funct. Anal. 233:2 (2006), 380-425. MR MR2214582

[Li and Li 2002] A. Li and Y. Li, "On some conformally invariant fully nonlinear equations", $C$. R. Math. Acad. Sci. Paris 334:4 (2002), 305-310. MR 2002m:35054 Zbl 0998.58011

[Li and Li 2003] A. Li and Y. Li, "Further results on Liouville type theorems for some conformally invariant fully nonlinear equations", 2003. math.AP/0301254

[Obata 1962] M. Obata, "Certain conditions for a Riemannian manifold to be isometric with a sphere”, J. Math. Soc. Japan 14 (1962), 333-340. MR 25 \#5479 Zbl 0115.39302

[Reilly 1973] R. C. Reilly, "On the Hessian of a function and the curvatures of its graph", Michigan Math. J. 20 (1973), 373-383. MR 48 \#12364 Zbl 0267.53003

[Viaclovsky 2000] J. A. Viaclovsky, "Conformal geometry, contact geometry, and the calculus of variations”, Duke Math. J. 101:2 (2000), 283-316. MR 2001b:53038 Zbl 0990.53035

Received August 25, 2004. Revised January 24, 2005.

MARÍA DEL MAR GONZÁlEZ

UNIVERSITY OF TEXAS AT AUSTIN

DEPARTMENT OF MATHEMATICS

1 UNIVERSITY STATION C1200

AUSTIN, TX 78712-0257

UNITED STATES

mgonzale@math.utexas.edu

http://www.ma.utexas.edu/users/mgonzale/ 